\title{
Counting of RBCs and WBCs in noisy normal blood smear microscopic images
}

\author{
M.Habibzadeh*a, A. Krzyżak ${ }^{\mathrm{a}}$, T.Fevens ${ }^{\mathrm{a}}$, A.Sadr ${ }^{\mathrm{b}}$ \\ ${ }^{a}$ Dept. of Computer Science and Software Engineering, Concordia University, Montréal, Canada \\ ${ }^{b}$ Dept. of Electrical Engineering, Sharif University, Tehran, Iran
}

\begin{abstract}
This work focuses on the segmentation and counting of peripheral blood smear particles which plays a vital role in medical diagnosis. Our approach profits from some powerful processing techniques. Firstly, the method used for denoising a blood smear image is based on the Bivariate wavelet. Secondly, image edge preservation uses the Kuwahara filter. Thirdly, a new binarization technique is introduced by merging the Otsu and Niblack methods. We have also proposed an efficient step-by-step procedure to determine solid binary objects by merging modified binary, edged images and modified Chan-Vese active contours. The separation of White Blood Cells (WBCs) from Red Blood Cells (RBCs) into two sub-images based on the RBC (blood's dominant particle) size estimation is a critical step. Using Granulometry, we get an approximation of the RBC size. The proposed separation algorithm is an iterative mechanism which is based on morphological theory, saturation amount and RBC size. A primary aim of this work is to introduce an accurate mechanism for counting blood smear particles. This is accomplished by using the Immersion Watershed algorithm which counts red and white blood cells separately. To evaluate the capability of the proposed framework, experiments were conducted on normal blood smear images. This framework was compared to other published approaches and found to have lower complexity and better performance in its constituent steps; hence, it has a better overall performance.
\end{abstract}

Keywords: CBC, RBC, WBC, Denoising, binarization, active contours, watershed

\section{INTRODUCTION}

The complete blood count (CBC) is used as a broad screening test and widely used diagnostic to check for such disorders as infections, allergies, problems with clotting, and for diagnosing and managing numerous diseases. It is actually a panel of tests that examines different particles of the blood drops and includes the WBC [1] and RBC [2] counts, WBC differential, sign of disease, and the number of infected cells. To do this, the blood film is stained $[3,4,5]$ (e. g., Wright's, Giemsa, or May-Grunwald) and then imaged with a transmission light microscope. The most reliable and complete diagnosis of blood smear infection is done by manually finding disorders and abnormalities in blood samples through a microscope, and counting blood smear particles. Not only is this a time consuming task but it is also subject to undesirable human error. In essence, the goal of this and related work is to develop and validate the necessary image processing steps to quantify and count peripheral blood particles on blood smear slides. The history of computerized research into automated blood slide examination dates back to 1975 with Bentley and Lewis [6]. The first jump to fully automated analysis of blood slides was presented by Rowan [7] in 1986. There is a large number of literature dedicated to differential blood counts, blood particles segmentation, blood classification and other related medical issue can be found in some sample works from 1976 up to 2010 [8-30].In essence, the current work aims to answer three questions: 1) how many primary particles such as RBCs and WBCs are present in a blood smear slide; 2) what is average size estimation of the RBC particles; and 3) how can we mitigate problems posed by different conditions such as noisy and degraded images, differing blood staining techniques, various types of microscope illumination, and overlapping and adjacent cells.

Medical Imaging 2011: Computer-Aided Diagnosis, edited by Ronald M. Summers, Bram van Ginneken, Proc. of SPIE Vol. 7963, 79633I - @ 2011 SPIE · CCC code: 0277-786X/11/\$18 - doi: 10.1117/12.878748 


\section{METHODS:}

This paper presents an accurate, robust mechanism to determine the distribution of blood smear particles. This work does not address issues such as deformed RBC shapes (teardrops, crescents, needles, or a variety of other forms), infected RBCs and extra overlapping cells which can be found with certain types of diseases.

Our method comprises nine steps: 1) Image acquisition and conversion to green channel. 2) De-noise with Bivariate Shrink Wavelet. 3) Edge Preserve with Kuwahara filter. 4) Binarization with Niblack \& Otsu. 5) Fill closed objects. 6) Size extraction. 7) Extract sub-image containing individual closed WBC region. 8) Separate WBCs from RBCs. 9) Counting algorithm. Details of each step are presented below.

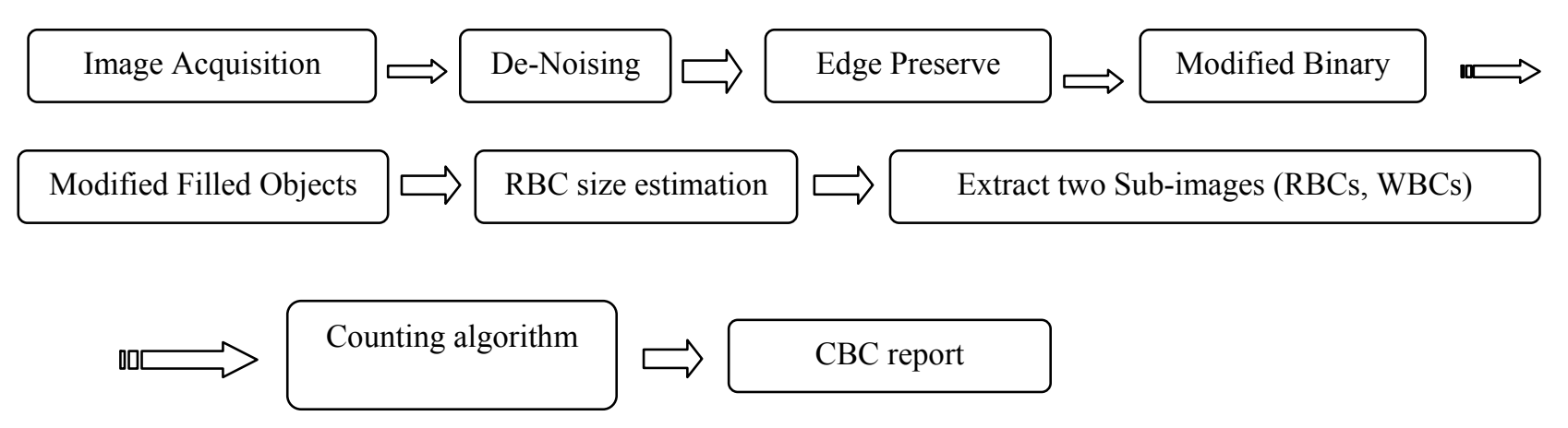

\subsection{Image acquisition and conversion to Green Channel:}

Our algorithm has been tested over sets of normal blood images collected from the GHODS polyclinic (Tehran, Iran) saved in JPEG format of size 512x512 pixels acquired using a Sony dfw-sx910 Camera. The first step is to convert images to the Green channel as it is more reliable than the Red or Blue channels for noisy and distorted images.

\subsection{Denoising with Bivariate Shrink Wavelet}

To design a reliable system that may be used under different conditions such as different blood staining techniques, types of chemical materials used, microscope types, illumination conditions, human errors, etc., a pre-processing step is required. Wavelet shrinkage is a signal denoising technique based on the idea of thresholding the wavelet coefficients of an image. For this work, Bivariate shrinkage based on Daubechies wavelets were used. From the experimental and mathematical results we concluded that for noisy microscopic images (even in the presence of high noise), the Bivariate Shrink filter [31,32] is optimal compared to other choices. It produces the maximum peak signal to noise ratio (PSNR) for the output image compared to the other filters considered However, the Bivariate output is blurred by default; hence, a recovery algorithm is required to smooth the texture while preserving edges.

\subsection{Edge Preservation with Kuwahara filter:}

Edge preservation is an image processing technique to recover degraded and blurred images while reducing the negative effect of noise in images. It can be a preliminary step toward better binarization and object segmentation. Different methods have been proposed: Median filter [33], Symmetrical Nearest Neighbor (SNN) filter [34], convolution kernel filters [35], preserving color reduction method [36], bilateral techniques [37], and the Kuwahara filter [38]. In this paper, the Kuwahara filter is used. Experimentation shows that because of intrinsic characteristics of blood smear particles, the Kuwahara filter has the sharpest edges which leads to better binarization in next step (see Fig. 2). However, the output may be somewhat toothy and jagged. 


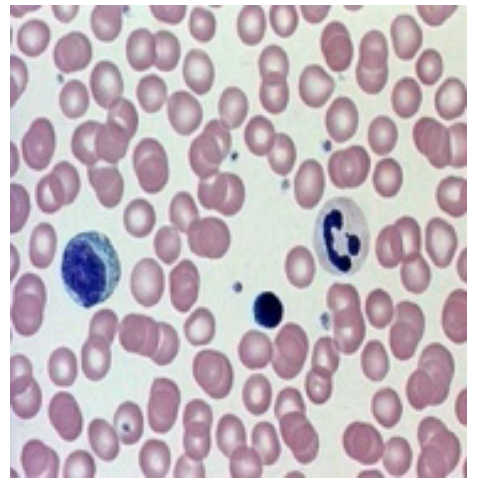

a)

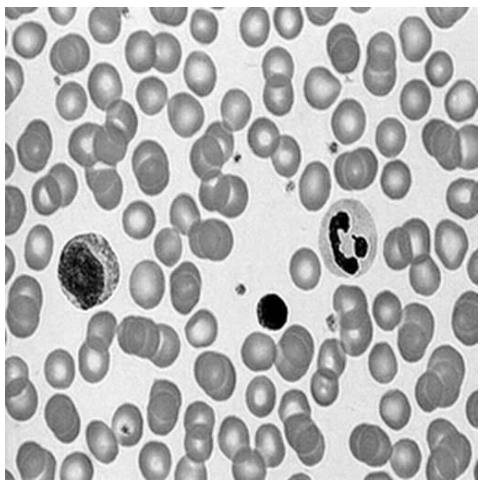

b)

Figure 1: (left to right): a) initial sample [39]; b) de-noised green channel of initial sample

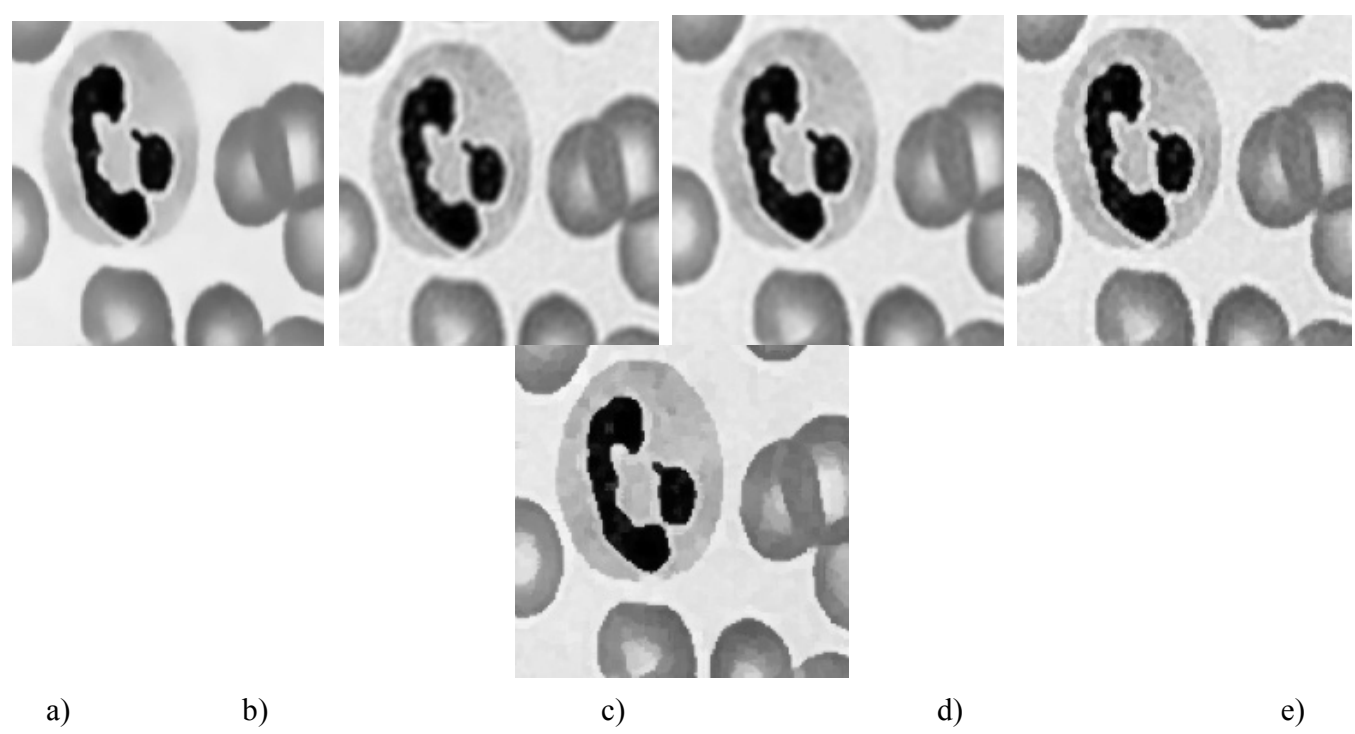

Figure 2: a) edge preservation with Bilateral, b) convolution kernel, c) EDGEPS [36],d) SNN, e) Kuwahara filters, respectively.

\subsection{Binarization with Niblack \& Otsu:}

After pre-processing (denoising and edge enhancement), binarization is the third step which allows to extract WBCs and RBCs sub-images, compute the cell sizes and count them. Generally, binarization methods can be applied with global and local thresholding. Different binarization methods include the approaches of Niblack [40], Bernsen [40], Sauvola [41] and Otsu [42]. In experiments over different samples with different initial conditions (see an example in Fig. 3) showed that Niblack is the most reliable method to maintain disjoint components which is crucial in avoiding over or under segmentation. 


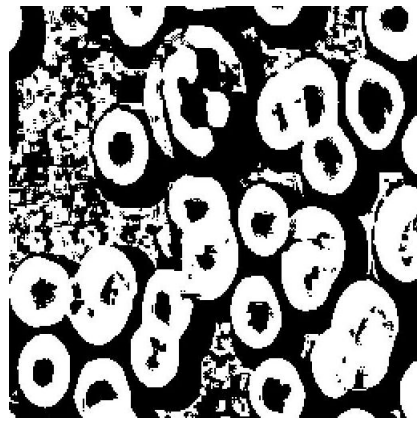

a)

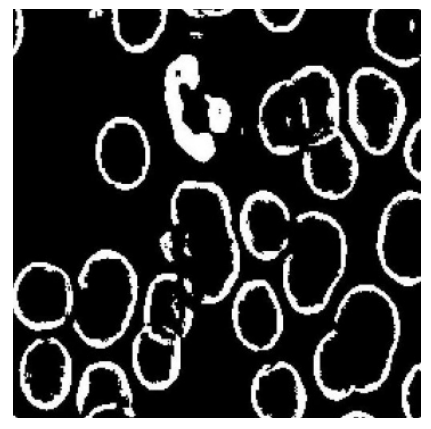

b)

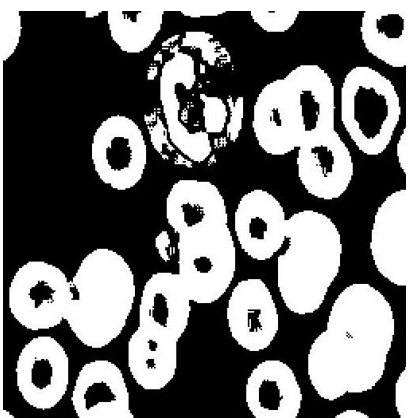

c)

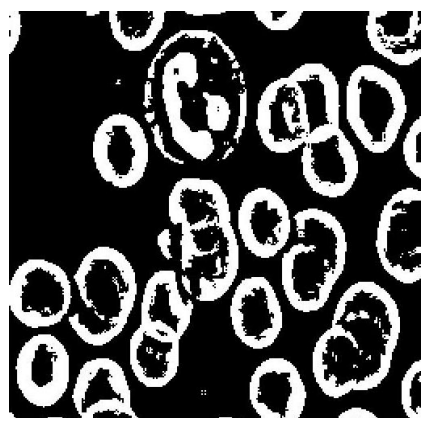

d)

Figure 3: Binarization methods: a) Bernsen; b) Sauvola; c) Otsu; and d) Niblack

In this work, a modified binarization method is proposed by merging Niblack and Otsu approaches. This process reduces limitations and drawbacks of each of them. In Niblack, there is a local thresholding based on $\mathrm{T}(\mathrm{x}, \mathrm{y})=\mathrm{m}(\mathrm{x}, \mathrm{y})+\mathrm{k} * \mathrm{~s}(\mathrm{x}, \mathrm{y})$ where $\mathrm{m}(\mathrm{x}, \mathrm{y})$ and $\mathrm{s}(\mathrm{x}, \mathrm{y})$ are the average and the standard deviation of a local area which the size of the window must be large enough to suppress the noise amount in the image as well as be also small enough to keep local details. In practice, a window size of 15-by-15 in all available database images works well. The "k" value helps to separate and adjust the percentage of pixels that belong to foreground (especially in the boundaries). We use $\mathrm{k}=-0.1$. Details of Otsu algorithm can be found in [42]. In some previously published papers [43], Otsu thresholding was the common binarization method; however, this method tends to result in overlapping objects that are too close to one another which in turn leads to false results after segmentation. In the modified version, pixels are labeled as backgrounds pixels if they are labeled as either background pixel in Niblack or in Otsu and the remaining points are kept as foreground (objects). Using this merging process, we mitigate the problem of extra small spurious regions produced by the Niblack algorithm.

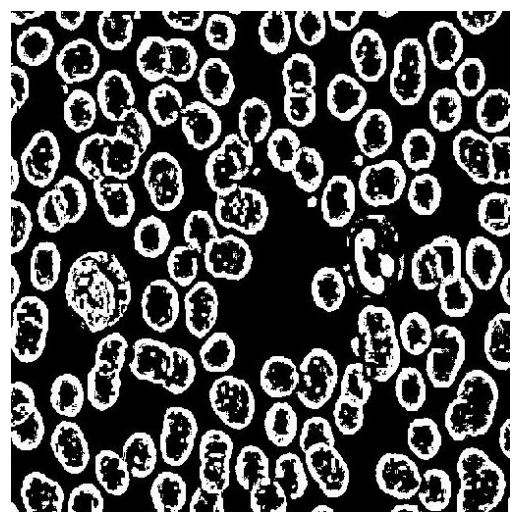

a)

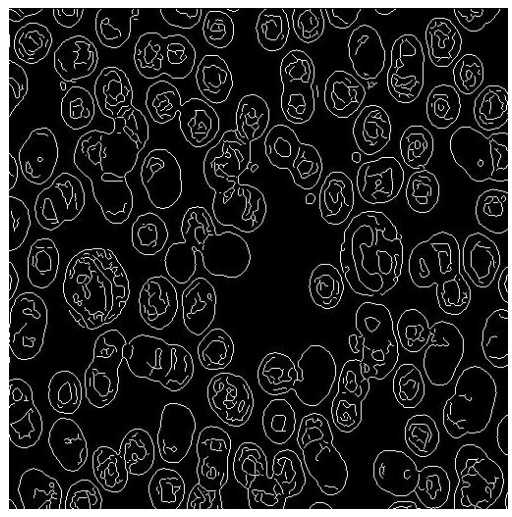

b)

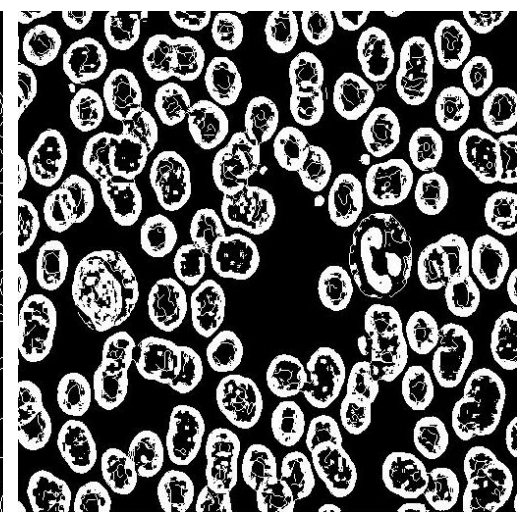

c)

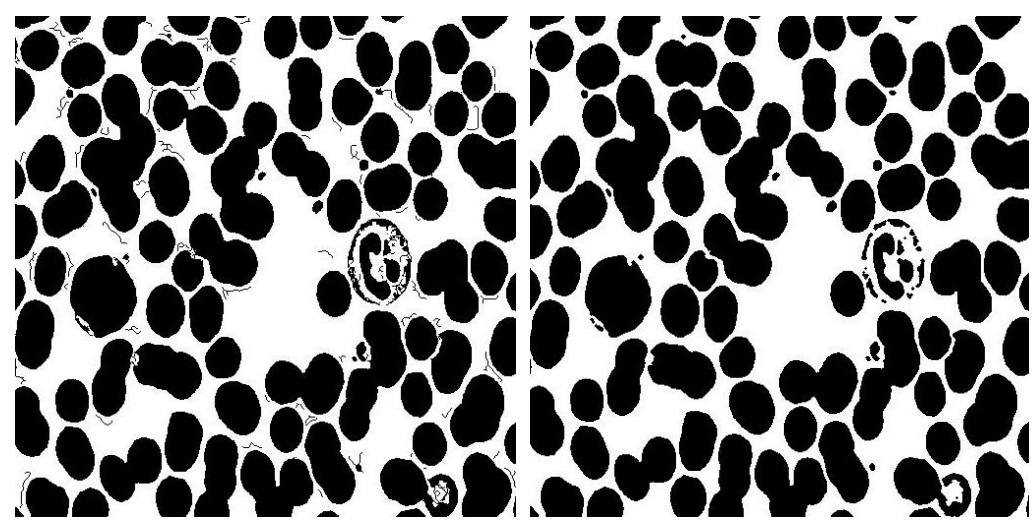


Figure 4: Edge detection and binarization: a) Modified binarization output, b) Canny edge detection, c)Merged binary and Canny outputs, d) Filled objects, e) Modified filled object (after applying closing transformation with SE=1px)

\subsection{Edge detection and binarization for hole filling:}

In the output of the modified binarization process, some RBCs and especially WBCs (due to their granular cytoplasm) may have boundaries that are not closed. Applying canny edge detection [44] (over the same edge preserving result of the Kuwahara filter) also gives an output that may boundaries that are not closed. Therefore, we merge the modified binarization and Canny output images to create an image to close the boundaries of RBCs and WBCs, whereupon the interiors of the closed boundaries are filled (see Fig. 4). Using the closing image transformation with defined SE (Structure Element) $=1 \mathrm{px}$ unwanted edges, typically generated by Canny, are removed.

\subsection{Size extraction:}

A normal blood cell is primarily one of two major particles: a RBC with a normal Probability Distribution Function (PDF) around 6.0-8.5 $\mu \mathrm{m}$ [2] or a WBC [45] $(7-18 \mu \mathrm{m})$ which includes a nucleus and cytoplasm is about 1-3 times bigger than normal and mature RBCs. Moreover, WBCs are classified into five main shape groups with varying degrees of non-convexity [46]. We use size characteristics as an effective factor to distinguish between the two main types of RBC and WBC cells. Granulometry [47] can determine the size distribution of image objects without explicitly segmenting each object first. According to normal Blood Probability Distribution Function (PDF) and RBC ratio in comparison with WBC numbers, the maximum regional peak in pattern spectrum diagram (Granulometry output; see Fig. 5) refers to the number of RBCs with an acceptable RBC radius size (in this sample is 10px). It is not possible, though, to estimate the size of WBCs based on granulometry because of their intrinsic characteristics and the overlapping.

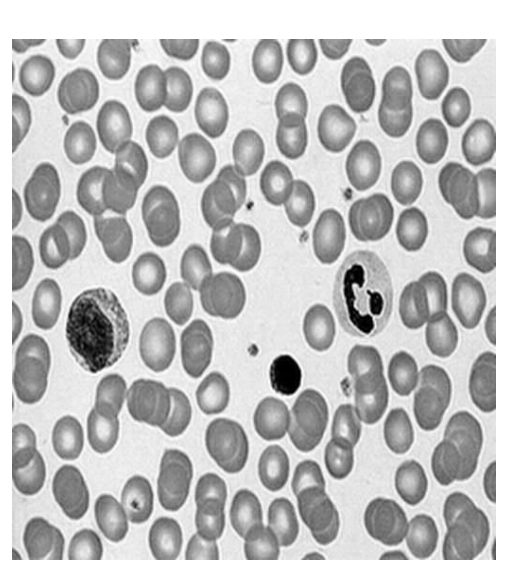

a)

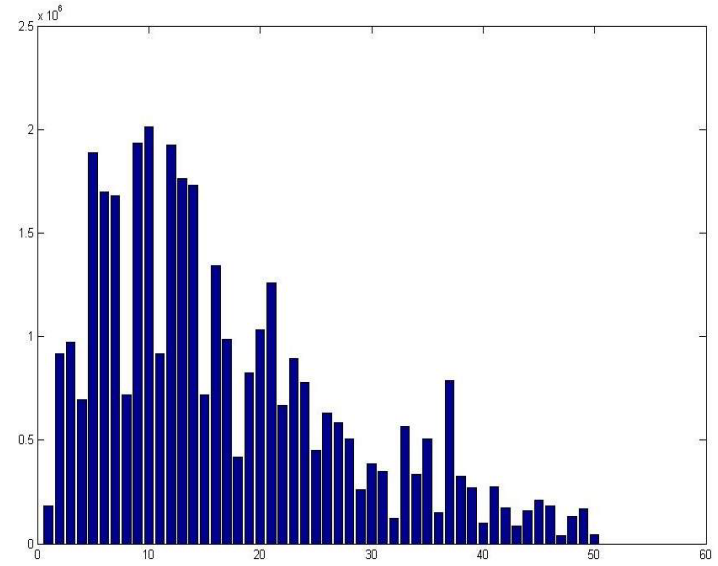

b)

Figure 5: Size extraction: a) de-noised green channel of initial sample; b) Granulometry over blood smear sample (RBC size detector)

\subsection{Extracting a sub-image containing individual closed WBC regions:}

First, a sub-image containing WBCs is separated from the image generated at the end of step 5) of the framework. This is done in five steps: A) An approximate location of nucleus is found by keeping $70 \%$ maximum "S" value in HSV channel module over edge preserved image. B) A morphological dilation by RBC size is performed over the discontinuous extracted dots (equal or greater than $70 \%$ maximum "S") to estimate and close the entire possible connective WBC region. C) A square mask surrounding the center of mass of connective regions (after dilation) with the size of $2 *$ diameter of mass region is applied over whole image to extract sub-images including separated WBCs and somewhat near RBCs. D) Since the boundaries of the WBCs in the image after merging binarization and canny output may still be imprecise, a more accurate estimation of the WBC boundaries can be obtained by applying an Active Contour using the Chan-Vese implementation [48] and then applying Canny edge detection to the resultant image. This edge -detected 
image is then merged with the image generated at the end of step 5 and the interiors of the cells are filled pixel-wise in this merged image. E) As a post-processing step some small spurious regions is cleaned up by using a closing morphological transformation (SE size $=1$ px). Example images of each step are shown in Fig. 6.

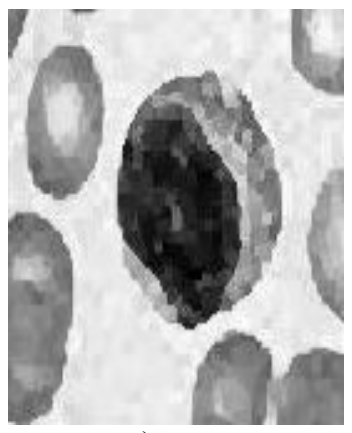

a)

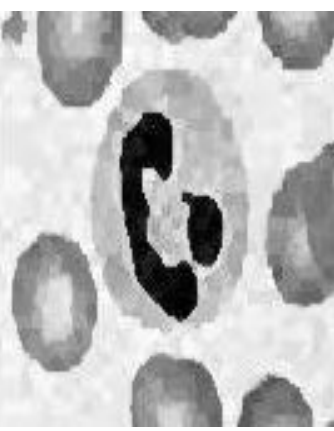

b)

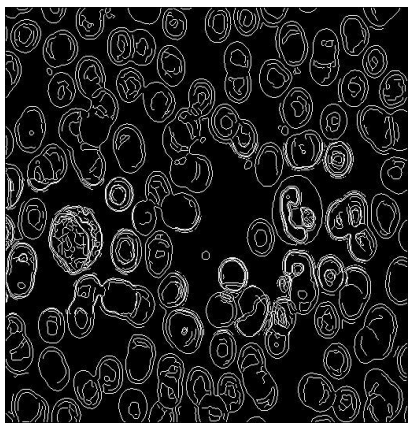

c)

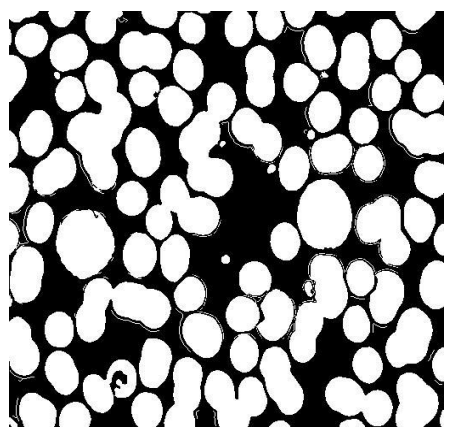

d)

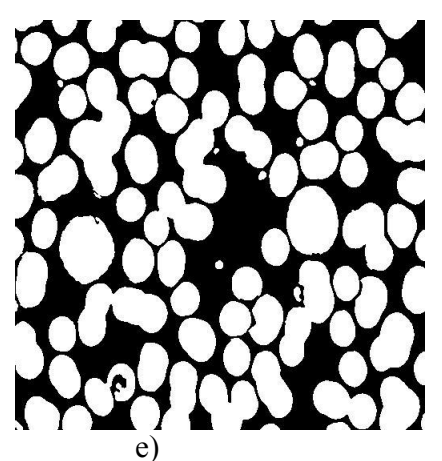

Figure 6: Extracting a sub-image containing individual closed WBC regions: a,b) sub-images containing WBCs; c) Canny over Chan-Vese Active Contour; d) adding new edged image and enhanced filled object; e) modified filled object (closing SE=1px)

\section{8. $\quad$ Separating WBCs from RBCs}

Thus far, an image is formed with solid objects; before counting, WBCs and RBCs should be separated into two subimages. This task could be done by a step- by-step iterative method: A) Apply granulometry over the blood smear image (with the RBC interiors filled in) and saving approximate RBC size. B) Initialize the possible available WBC size from expected physical characteristics and an acceptable marginal range: $\mathrm{C} 1=80 \% * \mathrm{RBC}$ size (as an initial marginal value). C) Moving the circular mask over blood smear image and detecting the exact matching objects of the same size. D) For those matched objects with any pixel with an $\mathrm{S}$ value greater than $70 \%$ of the maximum value (which indicates the presence of a nucleus here), all its pixel intensities are set to 0 (zero). E) Applying circular mask function in a closed loop by an initial radius value $(\mathrm{C} 1=80 \% *$ RBCsize $)$ and then moving the mask over all image pixels. F) Save the WBC indicator in a new image mask. G) Possible noisy remained region and speckles are removed by deleting closed objects less than $1 / 3 \mathrm{RBC}$ size. This procedure is time consuming but efficient for all five main kinds of WBCs. 


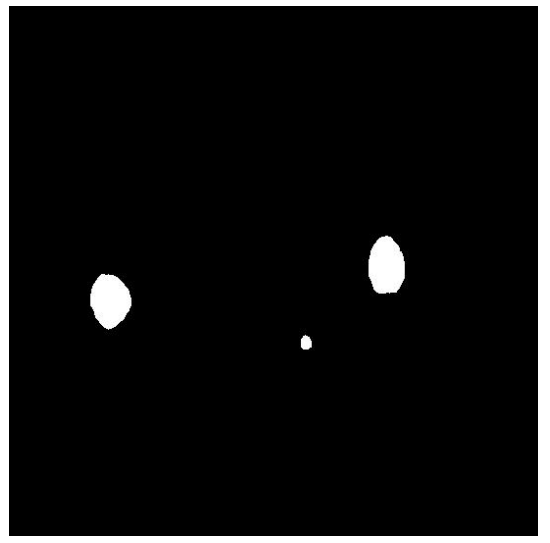

a)

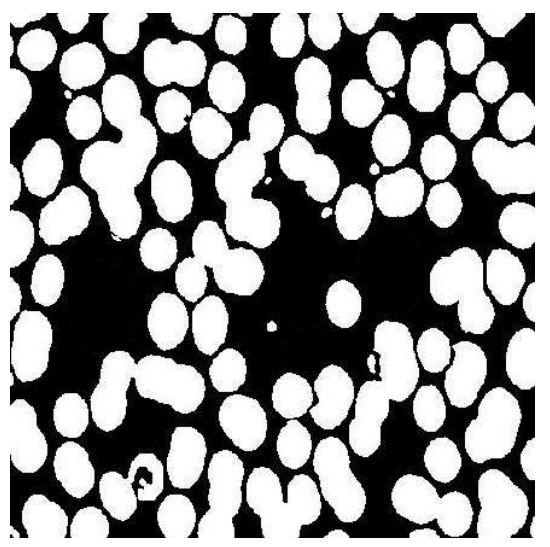

b)

Figure 7: Separating WBCs from RBCs: a) WBC indicator; b) Separated RBC sub-image

\subsection{Counting algorithm:}

To count objects in an image, watershed segmentation approaches [49] are frequently employed. In this work, the immersion watershed method is applied. The accuracy and efficiency of watershed segmentation over two blood smear sub-images is directly related to previous steps.

\section{RESULTS}

A set of blood smear test images were used to show that our proposed framework is more accurate in comparison with some classical methods, and also is much more robust for degraded images which are blurry and/or noisy. The first comparison method denoises the image with a median filter with a $3 \times 3$ mask function and then uses Otsu binarization to create an intermediate image. Then a second intermediate image is created using Canny edge detection. These two intermediate images are merged into the final image, objects with closed boundaries are filled pixelwise, and the number of objects is counted using watershed segmentation. The second comparison method performs the same procedure using Otsu binarization but skips the Canny edge detection step. In Table 1, 10 different blood smear slides (numbered N0 to N9) with a variety of image characteristics are segmented by our approach and compared with some known techniques such as different possible combination of Otsu, canny. In the last four rows, the images have had noise added to the images to test the robustness of our framework under extreme conditions. The results are compared with manual counts of the number of RBCs and WBCs, with the difference between the computed counts and the manual counts indicated by the numbers in parenthesis. The results show that our approach is much closer to the actual counts, especially in noisy images showing that our denoising techniques lead to better results. In particular, WBC counts are much more accurate with our framework than with those methods using different binarization techniques (a total of 1 miscounted, under or overcounted, WBC versus 76 for Otsu-Canny and 26 for Otsu for the 10 images without additive noise), while on the other hand, RBCs are frequently uncounted but to a smaller extent than the typical overcounts of the other techniques (a total of 65 miscounted RBCs versus 173 for Otsu-Canny and 140 for Otsu for images without additive noise). 
Table 1. Experimental results of 10 different blood smear images (N0-N9). Counts for RBCs and WBCs are given from manual counts, as determined by our framework, and as determined by two classical methods. Values given in parentheses are differences between counts computed and those obtained by a manual count (negative values indicate an undercount with respect to the manual count; positive values indicate an over count).

\begin{tabular}{|c|c|c|c|c|c|c|c|c|c|}
\hline \multirow{2}{*}{ 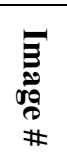 } & \multirow[b]{2}{*}{ Image Characteristics } & \multicolumn{2}{|c|}{$\begin{array}{c}\text { Manual } \\
\text { Count }\end{array}$} & \multicolumn{2}{|c|}{$\begin{array}{l}\text { Our Framework } \\
\text { Count (error) }\end{array}$} & \multicolumn{2}{|c|}{$\begin{array}{l}\text { Count using Otsu- } \\
\text { Canny Binarization }\end{array}$} & \multicolumn{2}{|c|}{$\begin{array}{c}\text { Count using Otsu } \\
\text { Binarization }\end{array}$} \\
\hline & & RBC & WBC & RBC & WBC & RBC & WBC & RBC & WBC \\
\hline N0 & Normal sample & 104 & 1 & $98(-6)$ & $1(0)$ & $122(18)$ & $9(8)$ & $115(11)$ & $4(3)$ \\
\hline N1 & Without WBCs & 75 & 0 & $70(-5)$ & $0(0)$ & $78(3)$ & $3(3)$ & $77(2)$ & $0(0)$ \\
\hline $\mathrm{N} 2$ & Blurred and overlapped & 135 & 2 & $132(-3)$ & $2(0)$ & $152(17)$ & $5(3)$ & $148(13)$ & $2(0)$ \\
\hline $\mathrm{N} 3$ & Image shown in Fig. 1 & 105 & 3 & $95(-10)$ & $3(0)$ & $122(17)$ & $12(9)$ & $128(23)$ & $9(6)$ \\
\hline N4 & Blurred & 335 & 1 & $330(-5)$ & $1(0)$ & $283(-52)$ & $44(43)$ & $295(-40)$ & $15(14)$ \\
\hline N5 & Blurred & 70 & 2 & $68(-2)$ & $2(0)$ & $90(20)$ & $1(-1)$ & $81(11)$ & $1(-1)$ \\
\hline N6 & Numerous overlapping & 90 & 2 & $76(-14)$ & $2(0)$ & $100(10)$ & $4(2)$ & $105(15)$ & $3(1)$ \\
\hline N7 & WBCs touch RBCs & 23 & 1 & $24(1)$ & $1(0)$ & $35(12)$ & $2(1)$ & $27(4)$ & $2(1)$ \\
\hline N8 & WBCs touch RBCs & 69 & 2 & $66(-3)$ & $2(0)$ & $81(12)$ & $5(3)$ & $80(11)$ & $2(0)$ \\
\hline N9 & $\begin{array}{l}\text { Blurred, numerous } \\
\text { overlapping, WBCs } \\
\text { touch RBCs }\end{array}$ & 120 & 1 & $104(-16)$ & $2(1)$ & $108(-12)$ & $4(3)$ & $110(-10)$ & $1(0)$ \\
\hline N6 & Additive high noise & 90 & 2 & $70(-20)$ & $3(1)$ & $12(-78)$ & $14(12)$ & $134(44)$ & $2(0)$ \\
\hline N9 & Additive high noise & 120 & 1 & $81(-39)$ & $2(1)$ & $56(-64)$ & $5(4)$ & $130(10)$ & $1(0)$ \\
\hline N6 & $\begin{array}{c}\text { Additive medium } \\
\text { noise }\end{array}$ & 90 & 2 & $74(-16)$ & $3(1)$ & $136(46)$ & $4(2)$ & $101(11)$ & $4(2)$ \\
\hline N9 & $\begin{array}{c}\text { Additive medium } \\
\text { noise }\end{array}$ & 120 & 1 & $86(-34)$ & $2(1)$ & $93(-27)$ & $4(3)$ & $96(-24)$ & 1 \\
\hline
\end{tabular}

\section{CONCLUSIONS}

In this paper, a simple and step-by-step efficient algorithm for noisy blood smear particles segmentation has been presented together with a new approach for fully automated detection and segmentation of RBCs and WBCs in a blood smear image. Experimental results indicate that the current analysis of blood cells is accurate and it offers remarkable segmentation accuracy. The performance of the proposed method is evaluated by comparing the automatically extracted blood particles with manual segmentations and other traditional techniques. Furthermore, the introduced method being simple and easy to implement is best suited for medical applications in clinical settings. The main breakthroughs are: (a) robust denoising by Bivariate shrinkage thresholding and mitigating its side effect problem (blurring) by using Kuwahara edge preservation filter; (b) introducing new calibrated binary images using merged Otsu and Niblack thresholding approach; and (c) separation of blood smear particles into two sub-images using a mask-based algorithm.

\section{ACKNOWLEDGMENT}

We would like to acknowledge the contributions of the following groups and individuals to the development of our paper and our presentation: Ms. Aida Habibzadeh, Ms.Parvaneh Saberian directed us to a wide range of resources on hematology science and also we appreciate GHODS polyclinic medical laboratory group cooperation to allow us using their equipments and provide the needed blood smear samples. 


\section{References}

1. S. M. Lewis, B. J. Bain, I. Bates, Dacie, Lewis "chapter 5:Blood cell morphology in health and disease" practical hematology, 10th ed. Philadelphia: Churchill Livingstone Elsevier, pp. 103,2006

2. S. M. Lewis, B. J. Bain, and I. Bates, Dacie and Lewis," chapter 5: Blood cell morphology in health and disease" practical hematology, 10th ed. Philadelphia: Churchill Livingstone Elsevier, pp. 80,2006.

3. Ramoser, H.; Laurain, V.; Bischof, H.; Ecker, R.; , Leukocyte segmentation and classification in bloodsmear images, Engineering in Medicine and Biology Society, 2005. IEEE-EMBS 2005. 27th Annual International Conference of the , vol., no., pp.3371-3374, 17-18 Jan. 2006

4. Joseph J. Schall, Professor. Ph.D , The University of Texas at Austin, "Making and Staining a Blood Smear" . uvm.edu. [Online]. Available:http://www.uvm.edu/ jschall/pdfs/techniques/bloodsmears.pdf. [Accessed: August. 06, 2010].

5. "Giemsa stain » .med-chem.com [Online]. Available: http://med-chem.com/procedures/Giemsabsp.pdf . [Accessed: august. 06, 2010].

6. Bentley, S. \& Lewis, S. The use of an image analyzing computer for the quantification of red cell morphological characteristics. British Journal of Hematology 29, 81-88. 1975

7. Rowan, R. Automated examination of the peripheral blood smear, in 'Automation and quality assurance in hematology', Blackwell Scientific, Oxford, chapter 5, pp. 129-177.1986

8. Martelli, A. An application of heuristic search methods to edge and contour detection Communications of the ACM 19(2), 73-83.1976

9. Pope, D., Parker, D., Clayton, P. \& Gustafson, D. Left ventricular border recognition using a dynamic search algorithm. Radiology 155(2), 513-518.1985

10. Fleagle, S.R.; Johnson, M.R.; Wilbricht, C.J.; Skorton, D.J.; Wilson, R.F.; White, C.W.; Marcus, M.L.; Collins, S.M.; Automated analysis of coronary arterial morphology in cineangiograms: geometric and physiologic validation in humans, IEEE Transactions on Medical Imaging, vol.8, no.4, pp.387-400, Dec 1989

11. Fleagle,S., Thedens,D., Ehrhardt,J., Scholz,T.\& Skorton,D. Automated identification of left ventricular borders from spin-echo magnetic resonance images. Investigative Radiology 26(4),295-303.1991

12. Adjouadi,M. ,Fernandez,N .An orientation-independent imaging technique for the classification of blood cells. Particle \& Particle Systems Characterization 18(2), 91-98.2001

13. Di Rubeto, C.; Dempster, A.; Khan, S.; Jarra, B.; Segmentation of blood images using morphological operators, Pattern Recognition, 2000. Proceedings. 15th International Conference on, vol.3, no., pp.397-400, 2000

14. Beucher, S. Watersheds of functions and picture segmentation, Acoustics, Speech, and Signal Processing, IEEE International Conference on ICASSP '82., vol.7, no., pp. 1928- 1931, May 1982

15. Beucher,S. The watershed transformation applied to image segmentation. Proceedings of the $10^{\text {th }}$ Pfefferkorn conference on Signal and Image Processing in Microscopy and Micro analysis, Cambridge, UK, pp 299-314. September 1991

16. M.Couprie, G. Bertrand. Topological grayscale watershed transformation. Proc. SPIE Vision Geometry V, vol. 3168, pp.136-146. 1997

17. Gauch, J.M.; Image segmentation and analysis via multi scale gradient watershed hierarchies, Image Processing, IEEE Transactions on, vol.8, no.1, pp.69-79, Jan 1999

18. C. De Roover, A. Herbulot, A. Gouze, E. Debreuve, M. Barlaud, and B. Macq, Multimodal segmentation combining active contours and watersheds, in Proceedings of the 13th European Signal Processing Conference (EUSIPCO '05), Antalya, Turkey, September 2005.

19. Kass, M., Witkin, A. \& Terzopoulos, D. Snakes: Active contour models. International Journal of Computer Vision 4, pp:321-331.1988

20. Leznray 0, Elmoataz A, Cardot H, Gougeon G, Lecluse M, Elie H, Revenu M. Segmentation of cytological images using color and mathematical morphology. European Congress of Stereology, 18(1): 1-14, 1999

21. Ongun, G.; Halici, U.; Leblebicioglu, K.; Atalay, V.; Beksac, M.; Beksac, S.; An automated differential blood count system, Engineering in Medicine and Biology Society, 2001. Proceedings of the 23rd Annual International Conference of the IEEE, vol.3, no., pp. 2583- 2586, 2001 
22. Ongun, G., Halici, U., Leblebicioglu, K., Atalay, V.Beksac, S. and Beksac, M. Automated contour detection in blood cell images by an efficient snake algorithm. Nonlinear Analysis-Theory Methods \& Applications 47(9), 5839-5847.2001

23. Ongun G, Halici U, Leblebicioglu K, Atalay V, Beksac M, Beksac S. Feature extraction and classification of blood cells for an automated differential blood count system. Proc. IJCNN, 4: 2461-2466. 2001

24. D. Comaniciu and P. Meer. Cell image segmentation for diagnostic pathology. In J. S. Suri, S. K. Setarehdan, and S. Singh, editors, advanced algorithmic approaches to medical image segmentation: state-of-the-art application in cardiology, neurology, mammography and pathology, pages 541-558. Springer. 2001

25. Kumar B R, Joseph D K, Sreenivas T V. Teager energy based blood cell segmentation. International Conference on Digital Signal Processing, 2: 619-622. 2002

26. N. Sinha and A. G. Ramakrishnan. Automation of differential blood count. In Proc. Conf. on Convergent Technologies for Asia-Pacific Region, volume 2, pages 547-551. 2003

27. K. Jiang, Q.-M. Liao and S.-Y. Dai. A novel white blood cell segmentation scheme using scale space filtering and watershed clustering. In Proc. Int. Conf. on Machine Learning and Cybernetics, volume 5, pages 28202825.2003

28. Theerapattanakul, J.; Plodpai, J.; Pintavirooj, C.; , An efficient method for segmentation step of automated white blood cell classifications, TENCON 2004. 2004 IEEE Region 10 Conference, vol.A, no., pp. 191- 194 Vol. 1, 21-24 Nov. 2004

29. Hamghalam, M.; Motameni, M.; Kelishomi, A.E.; , Leukocyte Segmentation in Giemsa-stained Image of Peripheral Blood Smears Based on Active Contour, 2009 International Conference on Signal Processing Systems , pp.103-106, 15-17 May 2009

30. F. Cloppet,A. Boucher. Segmentation of complex nucleus configurations in biological images. Pattern Recognition Letters, volume31, no:8,pages 755-761.2010

31. Sendur, L.; Selesnick, I.W.; Bivariate shrinkage functions for wavelet-based denoising exploiting interscale dependency, Signal Processing, IEEE Transactions on, vol.50, no.11, pp. 2744- 2756, Nov 2002

32. Sendur, L.; Selesnick, I.W.;A bivariate shrinkage function for wavelet-based denoising, Acoustics, Speech, and Signal Processing, 2002. Proceedings. (ICASSP '02). IEEE International Conference on, vol.2, no., pp.1261-1264, 2002

33. Lim, Jae S., Two-Dimensional Signal and Image Processing, Englewood Cliffs, NJ, Prentice Hall, pp. 469476, 1990.

34. Vinh Hong1, Henryk Palus2, and Dietrich Paulus. Edge Preserving Filters on Color Images. Springer-Verlag Berlin Heidelberg, vol.3039 no. pp.34-40, 2004.

35. Babic, Z.V.; Mandic, D.P.; An efficient noise removal and edge preserving convolution filter, Telecommunications in Modern Satellite, Cable and Broadcasting Service, 2003. TELSIKS 2003. 6th International Conference on , vol.2, no., pp. 538- 541 vol.2, 1-3 Oct. 2003

36. Nikos Nikolaou, Nikos Papamarkos. Color Reduction for Complex Document Images. International Journal of Imaging Systems and Technology, Vol. 19 (1) pp. 14-26, 2009.

37. Tomasi, C.; Manduchi, R.; bilateral filtering for gray and color images, Computer Vision, 1998. Sixth International Conference on, vol., no., pp.839-846, 4-7 Jan 1998

38. Papari, G.; Petkov, N.; Campisi, P.; Artistic Edge and Corner Enhancing Smoothing, Image Processing, IEEE Transactions on , vol.16, no.10, pp.2449-2462, Oct. 2007

39. "Connective tissue-blood \& blood forming tissues, http://www.rutgers.edu/,[online].available: http://lifesci.rutgers.edu/ babiarz/bloodtx.htm . [Accessed: August. 06, 2010].

40. Ntogas Nikolaos, Ventzas Dimitrios, A binarization algorithm for historical manuscripts, 12th WSEAS International Conference on communications, Heraklion, Greece, Pages: 41-51, 2008

41. Sauvola, M. Pietikainen. Adaptive document image binarization .Elsevier The journal of the pattern recognition society. Vol .33, Issue 2, pp:225-236, February 2000

42. Nobuyuki Otsu, A Threshold Selection Method from Gray-Level Histograms, Systems, Man and Cybernetics, IEEE Transactions on, vol.9, no.1, pp.62-66, Jan. 1979 
43. Bergen, Tobias; Steckhan, Dirk; Wittenberg, Thomas; Zerfass, Thorsten; Segmentation of leukocytes and erythrocytes in blood smear images, Engineering in Medicine and Biology Society, 2008. EMBS 2008. 30th Annual International Conference of the IEEE , vol., no., pp.3075-3078, 20-25 Aug. 2008

44. Canny, John ,A Computational Approach to Edge Detection, Pattern Analysis and Machine Intelligence, IEEE Transactions on, vol.PAMI-8, no.6, pp.679-698, Nov. 1986

45. S. M. Lewis, B. J. Bain, and I. Bates, Dacie and Lewis, chapter 5:Blood cell morphology in health and disease practical haematology, 10th ed. Philadelphia: Churchill Livingstone Elsevier, pp. 107-110, 2006

46. Newland J. The peripheral blood smear. Goldman L,Ausiello D, eds. Cecil Medicine. 23rd ed. Philadelphia, Pa: Saunders Elsevier, chap 161, 2007.

47. Luc Vincent. Fast Opening Functions and Morphological Granulometries. Image Algebra and Morphological Image Processing, SPIE Vol. 2300, pp. 253-267, July 1994

48. Chan, T.F.; Vese, L.A.; , Active contours without edges, Image Processing, IEEE Transactions on, vol.10, no.2, pp.266-277, Feb 2001

49. Yung-Chieh Lin; Yu-Pao Tsai; Yi-Ping Hung; Zen-Chung Shih; Comparison between immersion-based and toboggan-based watershed image segmentation, Image Processing, IEEE Transactions on, vol.15, no.3, pp.632-640, March 2006

50. Von Wegner, F.; Both, M.; Fink, R.H.A.; Friedrich, O.;Fast XYT Imaging of Elementary Calcium Release Events in Muscle With Multifocal Multiphoton Microscopy and Wavelet Denoising and Detection, Medical Imaging, IEEE Transactions on, vol.26, no.7, pp.925-934, July 2007

51. Negar M. Harandi, Saeed Sadri,Noushin A. Moghaddam, Rassul Amirfattahi. An Automated Method for Segmentation of Epithelial Cervical Cells in Images of ThinPrep. Springer Journal of medical systems. PP: 116,2009

52. Cecilia Di Ruberto, Andrew Dempster, Shahid Khan, Bill Jarra. Morphological Image Processing for Evaluating Malaria Disease. Springer-Verlag Berlin Heidelberg, Volume 2059, PP: 739-748, 2001. 